\title{
Effect of short-term physical exercise on serum markers of skeletal muscle damage in hypertensive patients treated with a combination of statin and angiotensin-converting enzyme inhibitor
}

\author{
Paweł Deska', Michał Nowicki \\ ${ }^{1}$ Department of Internal Medicine District Hospital, Kłobuck, Poland \\ ${ }^{2}$ Department of Nephrology, Hypertension and Kidney Transplantation, Medical University of Lodz, Łódź, Poland
}

\begin{abstract}
Background. Muscle strain during physical exercise may lead to muscle damage and hyperkalaemia. Commonly prescribed drugs including statins and angiotensin-converting enzyme inhibitors (ACEI) may increase serum potassium and cause muscle damage. We postulated that the risk may be even higher if the patients are treated with a combination of statin and ACEI.

The aim of the study was to compare the effect of moderate intensity short-term physical exercise on the changes of muscle injury markers serum carbonic anhydrase 3 and creatine kinase in patients with arterial hypertension receiving ACEI and statin.

Material and methods. Eighteen patients with arterial hypertension treated with ACEI were included in a prospective placebo-controlled cross-over study. 18 healthy individuals served as controls. Hypertensive patients underwent 30-minute exercise test, which was repeated two times in a random order after the administration of ACEI with statin or ACEI with placebo. Each treatment period lasted 8 weeks separated by 2 -week wash-out. Normotensive subjects underwent a single exercise test. Serum carbonic anhydrase 3 (CAIII) and creatine kinase (CK) activity were measured before and after exercise and recovery.

Results. At baseline and during the exercise CAIII was significantly higher in healthy volunteers compared to hypertensive patients. CAIII increased significantly during exercise only after treatment with both ACEI and statin. Serum CK was higher in hypertensive patients treated with ACEI plus statin during all exercise tests.

Conclusions. Addition of statin to ACEI in patients with arterial hypertension does not increase the risk of muscle damage during moderate intensity short-term physical exercise.

Key words: physical exercise; arterial hypertension; carbonic anhydrase III; creatine kinase; angiotensin-converting enzyme inhibitor; HMG-CoA reductase inhibitor
\end{abstract}




\section{Introduction}

Exercise-induced muscle damage is the consequence of a complex interactions of events, which seem to include muscle fibres damage due to myofibril injury (rupture of myofibrils), impaired excitation-contraction coupling associated with local ATP depletion, changes in the intracellular calcium homeostasis, oxidative stress and inflammation [1]. Vigorous physical exercise may affect the integrity of muscle cells and cause a release of muscle cell enzymes into the extracellular compartment. Muscle damage is a frequent event and many individuals are susceptible to muscle damage induced by physical exercise or caused by pharmacotherapy [2-4]. Serum concentration of creatine kinase and myoglobin are sensitive markers of muscle damage and myopathy $[5,6]$. Creatine kinase $(\mathrm{CK})$ is an enzyme expressed in large amounts in muscle cells. It catalyses the reversible process of transferring the phosphate group from phosphocreatine to adenosine diphosphate (ADP) thus rebuilding the adenosine diphosphate (ATP) inventory. The concentration of creatine kinase increases manifolds after the skeletal muscle damage caused by trauma, intramuscular drug injection, intense physical exercise, convulsions, myositis, muscular dystrophies and myotonia $[7,8]$.

Serum carbonic anhydrase III has been a well-established serum marker of skeletal muscle damage. Carbonic anhydrase 3 is a metalloenzyme found in the cytosol of a body cell. It catalyses the enzymatic reaction of hydrogen ion formation from water and carbon dioxide [9]. The enzyme was first detected in human skeletal muscle cells in 1979 [10-12]. Carbonic anhydrase 3 is selectively expressed in skeletal muscle, but not in the heart muscle, which was used in the differential diagnosis of stenocardia; however, a clinical interest in the routine measurement of this marker has diminished after the introduction of highly sensitive markers of myocardial ischaemia [13]. A significant increase in carbonic anhydrase 3 activity in the blood was reported after physical exercise and in several neuromuscular diseases that resulted in microdamage of muscle cells $[14,15]$.

Most patients with multiple coexisting diseases including arterial hypertension or coronary artery disease require an administration of statin. Taking into account a large proportion of patients treated with statins and the changes of lifestyle that have occurred in the modern societies aimed at increasing recreational physical activity including short-term and prolonged high-intensity exercises the potential risk of such adverse effects as hyperkalaemia and muscle damage of these drugs is becoming clinically relevant $[11,12]$.

The aim of the study was to assess the effect of a moderate intensity short-term standardized physical exercise on the changes of serum creatine kinase and carbonic anhydrase III activity in patients with arterial hypertension treated with an angiotensinconverting enzyme inhibitor alone or in a combination with statin.

\section{Material and methods}

In a prospective, cross-over and placebo-controlled study 18 patients with essential hypertension and 18 healthy individuals who served as a control group were included. The study design is presented in Figure 1.

The study protocol was approved by the Local Bioethics Committee. All subjects gave a written informed consent for the participation in the study.

The inclusion criteria were as follows: arterial hypertension diagnosed according to the $2013 \mathrm{ESH} /$ /ESC criteria [17], previously untreated or treated with maximum two antihypertensive drugs in therapeutic doses, women or men aged 18-70 years, serum potassium concentration less than $5.2 \mathrm{mmol} / \mathrm{l}$ at the time of screening, total cholesterol $>190 \mathrm{mg} / \mathrm{dL}$ or LDL-cholesterol $>115 \mathrm{mg} / \mathrm{dL}$.

The exclusion criteria included any secondary form of hypertension, motor organ disorders, inherited hypercholesterolemia, uncontrolled symptomatic heart failure (NYHA III and IV), uncontrolled arrhythmia, atrioventricular block $\mathrm{II}^{\circ}$ or $\mathrm{III}^{\circ}$, symptomatic aortic stenosis, history of myocarditis or pericarditis, acute infections, hyperthyroidism, chronic kidney disease stage $3 \mathrm{~A}$ or higher, i.e. estimated glomerular filtration rate calculated using the CKD-EPI [18] formula equal or smaller than $60 \mathrm{~mL} / \mathrm{min} / 1.73 \mathrm{~m}^{2}$, serum potassium higher than $5.5 \mathrm{mmol} / \mathrm{L}$ found at least twice at any time in the past, history of statin intolerance, uncontrolled diabetes mellitus, chronic liver disease, mental illness, history of non-compliance.

Exercise tests were conducted on a bicycle ergometer (Kettler Stratos, Ense-Parsit, Germany) following the same protocol in all participants. Each time, the exercise followed 20-minute preparation including the rest in a sitting position. A peripheral venous catheter was placed to withdraw blood for laboratory measurements. After a baseline blood sample was taken, the patients started the exercise that lasted 30 minutes with a load to achieve oxygen consumption in the range of $55-60 \%$ maximum based on the 


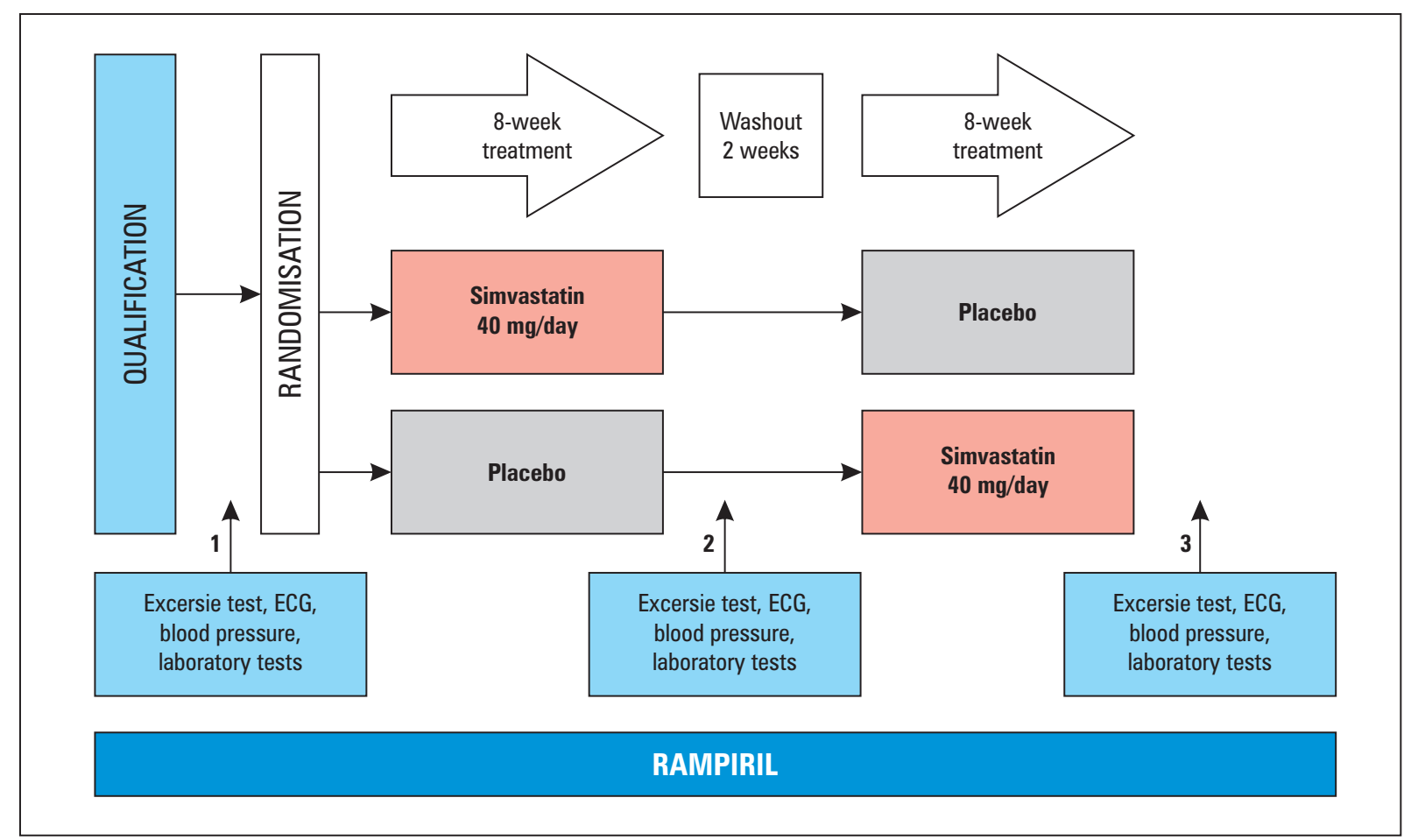

Figure 1. Study design and flowchart. ECG — electrocardiography

heart rate. After 15 and 30 minutes, blood samples were taken for laboratory tests. Upon the completion of exercise, the patients were asked to spend another 30 minutes in a sitting position to recover. After 30 minutes of the rest that followed the exercise the last blood sample was taken. During each examination the patients were asked to drink $500 \mathrm{~mL}$ of still lowmineralized water. The blood samples after the formation of blood clot were immediately centrifuged, and the serum was separated into separate test tubes frozen at $-80^{\circ} \mathrm{C}$ until laboratory measurements.

Creatine kinase was measured in plasma by the kinetic method using a Cobas Integra 400 Plus biochemical analyser (Roche, Munich, Germany).

Carbonic anhydrase 3 was measured in plasma by the immunoenzymatic method using the Cloud Clone Corp test. (Houston, USA).

All patients from the study group had been receiving a stable dose of ramipril $10 \mathrm{mg}$ each morning for at least 6 months before the study. The whole study lasted 18 weeks and was divided into 3 stages. After the first exercise test, the patients were randomly divided into two groups. An even parity of the numbers generated in MS Excel using RAND function was used for randomization.

During the first 8-week study period the patients received simvastatin in a dose of $40 \mathrm{mg}$ or placebo depending on the treatment order of the group. The drug and placebo were prepared by the hospital pharmacy in a form of tablets in identical appearance as the active drug. After 8 weeks of treatment, during which the study visits were held every 2 weeks the second exercise test was carried out following exactly the same scheme. The second test was followed by a wash-out period during which the study drug test or placebo were discontinued for two weeks. The wash-out period was followed by the third period lasting 8 weeks during which the patients received either drug or placebo. At the end of that period the patients underwent the third exercise test. The adherence to the therapy was verified by the counting of the packages and tablets of medicines returned by the patients at each visit. In a group of 18 healthy volunteers who were not taking any drugs the exercise test was carried out once according to the same protocol. The creatine kinase and carbonic anhydrase III activity were measured before each exercise test and immediately at its end and after a rest period.

Descriptive statistics included a calculation of the arithmetic mean and standard deviation (SD). Normality of the distribution of variables was examined with the Shapiro-Wilk test. Means of both groups were compared with two-sided t-test or Mann-Whitney test depending on the distribution of each variable. ANOVA was used to analyse repeated measurements. $\mathrm{p}<0.05$ was taken as statistically significant. 
Table I. Serum creatine kinase activity (before exercise, after 30-min exercise, after 30-min recovery)

\begin{tabular}{|l|c|c|c|}
\hline & $\begin{array}{c}\text { Before exercise } \\
{[\text { [IU/L] }}\end{array}$ & $\begin{array}{c}\text { After 30-min exercise } \\
{[\text { [UU/L] }}\end{array}$ & $\begin{array}{c}\text { After 30-min recovery } \\
{[\text { IU/L] }}\end{array}$ \\
\hline ACEI & $59 \pm 34$ & $59 \pm 27$ & $58 \pm 26$ \\
\hline ACEI + statin & $88 \pm 62$ & $94 \pm 51$ & $90 \pm 62$ \\
\hline ACEI + placebo & $62 \pm 32$ & $68 \pm 33$ & $68 \pm 27$ \\
\hline Control group & $48 \pm 24$ & $55 \pm 31$ & $51 \pm 30$ \\
\hline
\end{tabular}

ACEI — angiotensin converting enzyme inhibitor

Table II. Serum carbonic anhydrase 3 activity $(p<0.05)$, (before exercise, after 30-min exercise, after 30-min recovery)

\begin{tabular}{|l|c|c|c|}
\hline & $\begin{array}{c}\text { Before exercise } \\
{[\underline{\mathrm{IU}} / \mathrm{L}]}\end{array}$ & $\begin{array}{c}\text { After 30-min exercise } \\
{[\mathrm{IU} / \mathrm{L}]}\end{array}$ & $\begin{array}{c}\text { After 30-min recovery } \\
{[\mathrm{IU} / \mathrm{L}]}\end{array}$ \\
\hline ACEI & $15475 \pm 8304$ & $17326 \pm 11628$ & $19419 \pm 21159$ \\
\hline ACEI + statin & $18412 \pm 92556$ & $18101 \pm 12228$ & $26585 \pm 23537$ \\
\hline ACEI + placebo & $14619 \pm 9093$ & $15744 \pm 5736$ & $15419 \pm 9662$ \\
\hline Control group & $38122 \pm 24333$ & $32800 \pm 25163$ & $33699 \pm 34927$ \\
\hline
\end{tabular}

ACEI - angiotensin converting enzyme inhibitor

\section{Results}

All initially qualified patients completed the study. None of participants reported any adverse reaction to the study drugs that led to the withdrawal of the treatment. Serum creatine kinase and carbonic anhydrase III activity both at the rest and during the physical exercise remained within the normal range in both groups.

The results of serum creatine kinase activity measurements are shown in Table I. There were significant differences in serum creatine kinase activity between the patients receiving ACEI and statin and patients treated only with ACEI or healthy volunteers at baseline and during the exercise test and after recovery $(\mathrm{p}<0.05)$. The serum creatine kinase activity was higher in hypertensive patients treated ACEI combined with statin compared to the patients treated with ACEI only or healthy volunteers. There were no significant changes of creatinine kinase during the exercise in each group.

The carbonic anhydrase 3 serum activity is shown in Table II. The activity was significantly higher in the control group compared to the hypertensive patients treated with ACEI or with both ACEI and statin with the exception of ACEI plus statin group after 30-minute recovery where the difference was not significant $(\mathrm{p}<0.05)$.

Figure 2 shows the percentage changes of carbonic anhydrase activity 3 during the short-term physical exercise in all parts of the study. The largest increase in the carbonic anhydrase 3 activity was found in patients treated with both ramipril and simvastatin.
In the control group, the carbonic anhydrase 3 activity decreased during the exercise but later after the recovery a small nominal increase was noted.

\section{Discussion}

The main result of our study is the demonstration of the lack of an increased risk of muscle damage after the administration of the combination of statin and ACEI in hypertensive patients during a moderate short-term physical exercise.

During the treatment with statin, muscle damage was frequently reported, manifested mostly by a muscle pain associated with modest or mild increase in the serum markers of muscle damage, e.g. serum creatine kinase [19-21]. No clinical symptoms that might have suggested statin-induced muscle damage were seen in our patients during the study and the physical exercise was very well-tolerated.

Previously the incidents of the severe complication of statin therapy including rhabdomyolysis and severe hyperkalaemia and renal failure have also been reported [16, 22]. A rhabdomyolysis was described mostly after a mechanical muscle damage including intramuscular injections leading to muscle damage [23].

Post-exercise muscle damage usually does not result in any relevant clinical consequences and remains subclinical but could be detected as an increase in specific serum markers of muscle damage determined in laboratory tests. Only in extreme cases it can lead to rhabdomyolysis. Rhabdomyolysis after 


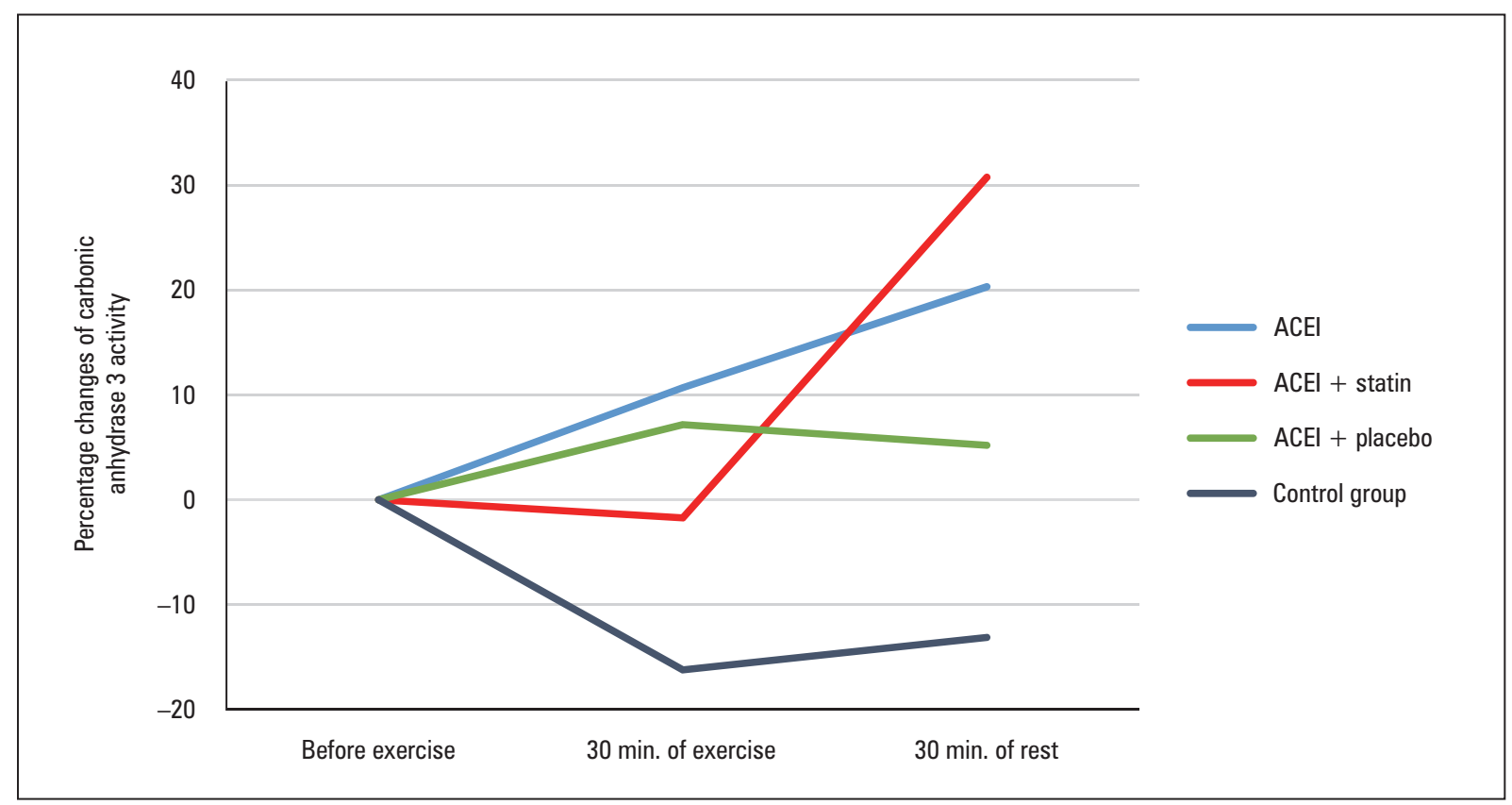

Figure 2. Percentage changes of carbonic anhydrase 3 activity from baseline during the exercise test. ACEI — angiotensin converting enzyme inhibitor

physical exercise was also reported in healthy athletes not taking any drugs and in the amateurs taking part in recreational sport activities. Most of the affected subjects were marathon runners, cyclists and those practicing other exhausting disciplines in unfavourable weather conditions [24-27]. However, the information about the risk of such events in a general hypertensive population is missing. Therefore we were not able to directly compare our results with the results of other authors.

The choice of a particular statin for the study also needs to be discussed. In our study, simvastatin in a dose of $40 \mathrm{mg} /$ day was used and the treatment duration was 8 weeks. We choose simvastatin for the study due to its common use in our country [28]. Simvastatin has a good safety profile and does not need to be reduced in mild renal function impairment that is commonly seen in hypertensive patients.

In order to evaluate the muscle damage each participant was first asked about the history of episodes of muscle weakness and myalgia and unexplained changes in urine colour. It has been reported that such symptoms may occur in 7-29\% of statin-treated patients, although most often in those receiving the highest recommended doses of the drug [29].

Muscle cell damage can lead to a release of numerous substances specifically expressed in the skeletal muscle cells into the blood, including ions and en- zymes. Among them such markers as creatine kinase, myoglobin and skeletal muscle-specific carbonic anhydrase III have been best studied [14, 15].

The mean creatine kinase activity remained within the normal range in our patients during the whole study. Despite that we found that creatine kinase activity in hypertensive patients was significantly higher in patients treated with ramipril combined with simvastatin when compared to the treatment with ACEI alone. This may suggest the effect of statins on the subclinical muscle cell damage. The lack of any significant increase in muscle damage markers in hypertensive patients who received only an ACEI may suggest that the effect could be attributed only to a statin. We have also looked at the post-exercise changes of muscle damage markers and we found a small non-significant numerical increase in these markers during the recovery from exercise. Even small changes in creatine kinase activity during exercise seem to confirm the effect of exercise on the integrity of muscle cells, which is consistent with earlier studies performed by other authors but in different populations and different experimental conditions [30, 31].

The determination of serum creatine kinase activity alone, although common in clinical practice may be insufficient since often even in patients reporting the symptoms indicative of myalgia, there is no detectable increase in the serum activity of this enzyme [32]. That is why we decided to determine also the 
activity of carbonic anhydrase 3 that is a more selective and sensitive marker of muscle damage $[15,33]$.

In the available literature a significant increase in carbonic anhydrase 3 activity was described in several conditions leading to overload and muscle damage $[14,15]$.

To maximize the safety of the patients, we decided to choose the intensity and duration of physical exercise that would be well tolerated by the patients and remain in a range mimicking recreational physical activity. Each time during the study, the participants were asked to drink $500 \mathrm{ml}$ of low-mineralized water for proper hydration in order to maintain adequate diuresis and reducing the risk of dehydration [34].

Despite the small group size the study had a crossover design to increase the statistical power by allowing the intra-group comparisons, as each patient received both study drugs and a placebo. The subjects were also carefully selected to ensure the homogeneity of the population.

The low number of study participants was also the result of long study protocol, which included at least a six-month period of ramipril treatment at a dose of $10 \mathrm{mg} / 24 \mathrm{~h}$ before the start of exercise tests, and then a total of ten-week treatment after randomisation. For safety reason we excluded the patients with chronic kidney disease stage $3 \mathrm{a}$ and higher. In such patients, the expected risk of complications including severe hyperkalaemia resulting from renal failure may have been much higher reported as high as $50 \%$ in some studies [35]. In addition, the patients with a creatinine clearance below $60 \mathrm{~mL} / \mathrm{min} / \mathrm{m}^{2}$ require a reduction of the dose of ramipril and or statin, which could hinder the interpretation of our results.

Our results may have some implications for clinical practice in frequent cases of the treatment of hypertensive patients with the combination of ACEI and statin. Our results suggest that such the combination has a favourable safety profile.

\section{Conclusions}

Moderate physical exercise in hypertensive patients treated with an angiotensin-converting enzyme inhibitor in combination with statin seems to be safe and does not increase the risk of clinically significant muscle damage.

\section{References}

1. Nikolaidis M, Jamurtas A, Paschalis V, et al. The Effect of MuscleDamaging Exercise on Blood and Skeletal Muscle Oxidative Stress. Sports Med. 2008; 38(7): 579-606, doi: 10.2165/00007256200838070-00005.
2. Siracusa J, Koulmann N, Sourdrille A, et al. Phenotype-Specific Response of Circulating miRNAs Provides New Biomarkers of Slow or Fast Muscle Damage. Front Physiol. 2018; 9: 684, doi: 10.3389/ fphys.2018.00684, indexed in Pubmed: 29922177.

3. Tomaszewski M, Stępień KM, Tomaszewska J, et al. Statin-induced myopathies. Pharmacol Rep. 2011; 63(4): 859-866, doi: 10.1016/ s1734-1140(11)70601-6, indexed in Pubmed: 22001973.

4. Mammen A. Statin-Associated Autoimmune Myopathy. N Engl J Med. 2016; 374(7): 664-669, doi: 10.1056/nejmra1515161, indexed in Pubmed: 26886523.

5. Lee G. Exercise-induced rhabdomyolysis. R I Med J (2013). 2014; 97(11): 22-24, indexed in Pubmed: 25365815.

6. Fitzgerald K, Redmond E, Harbor C. Statin-induced Myopathy. Glob Adv Health Med. 2012; 1(2): 32-36, doi: 10.7453/ gahmj.2012.1.2.008, indexed in Pubmed: 24278816.

7. Cervellin G, Comelli I, Benatti M, et al. Non-traumatic rhabdomyolysis: Background, laboratory features, and acute clinical management. Clin Biochem. 2017; 50(12): 656-662, doi: 10.1016/j. clinbiochem.2017.02.016, indexed in Pubmed: 28235546.

8. Lott JA, Stang JM. Serum enzymes and isoenzymes in the diagnosis and differential diagnosis of myocardial ischemia and necrosis. Clin Chem. 1980; 26(9): 1241-1250, indexed in Pubmed: 6994925.

9. Monti DM, De Simone G, Langella E, et al. Insights into the role of reactive sulfhydryl groups of Carbonic Anhydrase III and VII during oxidative damage. J Enzyme Inhib Med Chem. 2017; 32(1): 5-12, doi: 10.1080/14756366.2016.1225046, indexed in Pubmed: 27766895

10. Harju AK, Bootorabi F, Kuuslahti M, et al. Carbonic anhydrase III: a neglected isozyme is stepping into the limelight. J Enzyme Inhib Med Chem. 2013; 28(2): 231-239, doi: 10.3109/14756366.201 2.700640, indexed in Pubmed: 22803676.

11. Beuerle JR, Azzazy HM, Styba G, et al. Characteristics of myoglobin, carbonic anhydrase III and the myoglobin/carbonic anhydrase III ratio in trauma, exercise, and myocardial infarction patients. Clin Chim Acta. 2000; 294(1-2): 115-128, doi: 10.1016/s00098981(99)00261-2, indexed in Pubmed: 10727678.

12. Deska P, Nowicki M. Short-term changes of serum potassium concentration induced by physical exercise in patient with arterial hypertension treated with angiotensin-converting enzyme inhibitor alone or in combination with statin. J Physiol Pharmacol. 2017; 68(1): 133-138, indexed in Pubmed: 28456777.

13. Feng HZ, Jin JP. Carbonic Anhydrase III Is Expressed in Mouse Skeletal Muscles Independent of Fiber Type-Specific Myofilament Protein Isoforms and Plays a Role in Fatigue Resistance. Front Physiol. 2016; 7: 597, doi: 10.3389/fphys.2016.00597, indexed in Pubmed: 28018233.

14. Väänänen $H K$, Syrjälä $H$, Rahkila $P$, et al. Serum carbonic anhydrase III and myoglobin concentrations in acute myocardial infarction. Clin Chem. 1990; 36(4): 635-638, indexed in Pubmed: 2108824.

15. Osterman PO, Askmark H, Wistrand PJ. Serum carbonic anhydrase III in neuromuscular disorders and in healthy persons after a long-distance run. J Neurol Sci. 1985; 70(3): 347-357, doi: 10.1016/0022510x(85)90176-5, indexed in Pubmed: 3932601.

16. Laufs U, Scharnagl H, Halle M, et al. Treatment Options for Statin-Associated Muscle Symptoms. Dtsch Arztebl Int. 2015, doi: 10.3238/arztebl.2015.0748

17. Guidelines for the management of arterial hypertension. www. escardio.org (10.1159/isbn.978-3-318-01088-6).

18. KDIGO 2012 Clinical Practice Guideline for the Evaluation and Management of Chronic Kidney Disease. Kidney Int. 2013; 3(1): Suppl.

19. Manoj K, Jain N, Madhu SV. Myopathy in Patients Taking Atorvastatin: A Pilot Study. Indian J Endocrinol Metab. 2017; 21(4): 504-509, doi: 10.4103/ijem.IJEM_79_17, indexed in Pubmed: 28670530

20. Khine H, Yuet WC, Adams-Huet B, et al. Statin-associated muscle symptoms and SLCO1B1 rs4149056 genotype in patients with familial hypercholesterolemia. Am Heart J. 2016; 179: 1-9, doi: 10.1016/j.ahj.2016.05.015, indexed in Pubmed: 27595674. 
21. Allen SC, Mamotte CDS. Pleiotropic and Adverse Effects of Statins-Do Epigenetics Play a Role? J Pharmacol Exp Ther. 2017; 362(2): 319-326, doi: 10.1124/jpet.117.242081, indexed in Pubmed: 28576976.

22. Osaki Y, Nakagawa Y, Miyahara S, et al. Skeletal muscle-specific HMG-CoA reductase knockout mice exhibit rhabdomyolysis: A model for statin-induced myopathy. Biochem Biophys Res Commun. 2015; 466(3): 536-540, doi: 10.1016/j.bbrc.2015.09.065, indexed in Pubmed: 26381177.

23. Muscal E. Rhabdomyolysis: Practice Essentials, Background, Pathophysiology. Medscape 2015. https://emedicine.medscape.com/ article/1007814-overview\#showall.

24. Gautam PL, Luthra N, Nain S. Sports Induced Cardiac Arrest: A Case of Missed Rhabdomyolysis. J Clin Diagn Res. 2015; 9(9): UD01-UD02, doi: 10.7860/JCDR/2015/10275.6507, indexed in Pubmed: 26500986.

25. Tunstall Pedoe DS. Marathon cardiac deaths: the London experience. Sports Med. 2007; 37(4-5): 448-450, doi: 10.2165/00007256200737040-00046, indexed in Pubmed: 17465632.

26. Rae DE, Knobel GJ, Mann T, et al. Heatstroke during endurance exercise: is there evidence for excessive endothermy? Med Sci Sports Exerc. 2008; 40(7): 1193-1204, doi: 10.1249/ MSS.0b013e31816a7155, indexed in Pubmed: 18580397.

27. Sinert R, Kohl L, Rainone T, et al. Exercise-induced rhabdomyolysis. Ann Emerg Med. 1994; 23(6): 1301-1306, doi: 10.1016/s01960644(94)70356-6, indexed in Pubmed: 8198305.

28. Sliż D, Filipiak KJ, Naruszewicz M, et al. Standards of statin usage in Poland in high-risk patients: 3ST-POL study results. Kardiol Pol. 2013; 71(3): 253-259, doi: 10.5603/KP.2013.0037, indexed in Pubmed: 23575780.
29. Stroes ES, Thompson PD, Corsini A, et al. European Atherosclerosis Society Consensus Panel. Statin-associated muscle symptoms: impact on statin therapy-European Atherosclerosis Society Consensus Panel Statement on Assessment, Aetiology and Management. Eur Heart J. 2015; 36(17): 1012-1022, doi: 10.1093/eurheartj/ehv043, indexed in Pubmed: 25694464.

30. Kim M, Chun J, Jung HAh, et al. Capillarisin attenuates exercise-induced muscle damage through MAPK and NF- $\kappa$ B signaling. Phytomedicine. 2017; 32: 30-36, doi: 10.1016/j.phymed.2017.04.007, indexed in Pubmed: 28732805.

31. Callegari GA, Novaes JS, Neto GR, et al. Creatine Kinase and Lactate Dehydrogenase Responses after Different Resistance and Aerobic Exercise Protocols. J Hum Kinet. 2017; 58: 65-72, doi: 10.1515/ hukin-2017-0071, indexed in Pubmed: 28828078.

32. Laufs U, Filipiak KJ, Gouni-Berthold I, et al. SAMS expert working group. Practical aspects in the management of statinassociated muscle symptoms (SAMS). Atheroscler Suppl. 2017; 26: 45-55, doi: 10.1016/S1567-5688(17)30024-7, indexed in Pubmed: 28434484.

33. Vasilaki A, Simpson D, McArdle F, et al. Formation of 3-nitrotyrosines in carbonic anhydrase III is a sensitive marker of oxidative stress in skeletal muscle. Proteomics Clin Appl. 2007; 1(4): 362-372, doi: 10.1002/prca.200600702, indexed in Pubmed: 21136689.

34. Muscal E. Rhabdomyolysis: Practice Essentials, Background, Pathophysiology. Medscape 2015. https://emedicine.medscape.com/ article/1007814-overview\#showall.

35. Sarafidis PA, Blacklock R, Wood E, et al. Prevalence and factors associated with hyperkalemia in predialysis patients followed in a low-clearance clinic. Clin J Am Soc Nephrol. 2012; 7(8): 1234-1241, doi: 10.2215/CJN.01150112, indexed in Pubmed: 22595825. 